\title{
A new formula for the optimum width of Substrate Integrated Waveguide
}

\author{
*Nassir Guellil ${ }^{1}$, Farid Djahli ${ }^{1}$, Chemseddine Zebiri² \\ ${ }^{1}$ LIS laboratory, Electronics department, Ferhat Abbas University of Setif 1, Setif, Algeria \\ ${ }^{2}$ LEPCI laboratory, Electronics department, Ferhat Abbas University of Setif 1, Setif, Algeria \\ *corresponding author, E-mail: guellilnassir@yahoo.fr
}

\begin{abstract}
A new formula for calculating the optimum width of a Substrate Integrated Waveguide (SIW) corresponding to the first mode is presented in this paper. Finite Difference Frequency Domain (FDFD) method is applied to analyze the waveguide structure where geometrical parameters of the SIW are iteratively varied in order to minimize the gap between cutoff frequencies of SIW structure and that of an equivalent conventional rectangular waveguide. Adequate parameters are used to derive the new formula. To verify the accuracy of the new formula, several waveguides are designed and analyzed using the commercial software HFSS. The calculated propagation constants are compared with experimental measurements from literature, a very good conformity is obtained.
\end{abstract}

\section{Introduction}

Substrate Integrated Waveguide (SIW) technology represents henceforth an important part of planar circuits. It consists of a metalized substrate on upper and lower sides, metal holes of diameter $d$ and longitudinal centre-to-centre spacing $p$ to form two rows that reduce lateral radiation losses (Figure 1). The cage structure appears to be a conventional rectangular waveguide. This technology can largely preserve the advantages of the classical rectangular waveguide (such high-quality factor and low-loss by radiation), and the advantages of planar circuits (simplicity of realization, low cost and high integration with the active components on the same substrate). Due to the advantages of this new technology, a variety of microwave components such as couplers, isolators and phase shifters are designed and marketed for different frequency bands. To have the same propagation and dispersion characteristics of a conventional rectangular waveguide, the design of SIW starts by determining the equivalent waveguide width for the desired cutoff frequency and a chosen substrate permittivity. For this reason, the equivalent width has a capital importance in the design of SIW circuits. To determine SIW's equivalent waveguide width, several formulas have been proposed in the literature. The most popular formula is presented in [1], where the BI-RME method is used to calculate admittance matrix of a periodic cell. correspondence between the cutoff frequency and SIW's width is derived. In reference [2], a Mode-Matching Technique (MMT) is used, where a circular-to-square via conversion is employed. The optimal width is obtained by minimizing the reflection between an equivalent waveguide and SIW structure. Authors of [3] have employed an approach based on a Method of Moment (MoM), where the equivalence on propagation and cutoff frequency between SIW and rectangular waveguide are investigated to find an equation which gives the equivalent width. Finally, an empirical formula giving the normalized width of the equivalent waveguide is presented in [4]. In this paper, we present a new formula for calculating SIW's optimum width in function of equivalent width $W e q$ and via diameter to via pitch ratio $(d / p)$. The Finite Difference Frequency Domain (FDFD) method, implemented in Matlab, is applied to extract the propagation characteristics of the SIW structure. Note that the propagation characteristic accuracy of SIW structure is an important criterion in any successful design of SIW-based circuits.

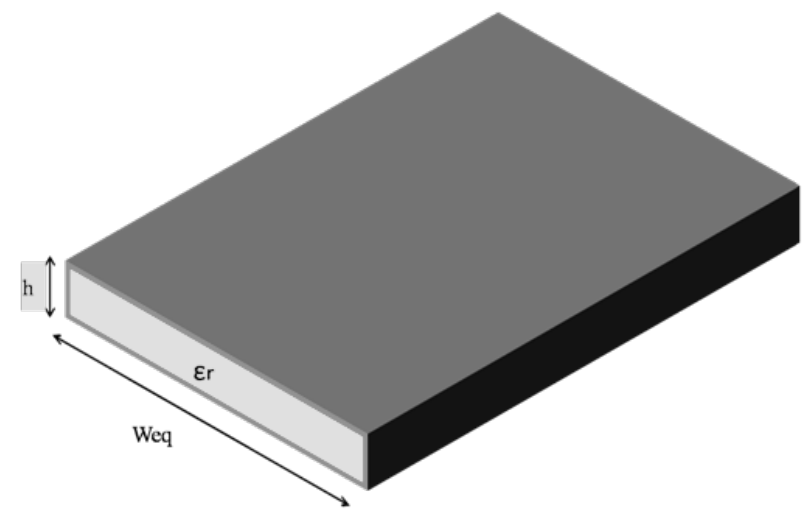

(a)

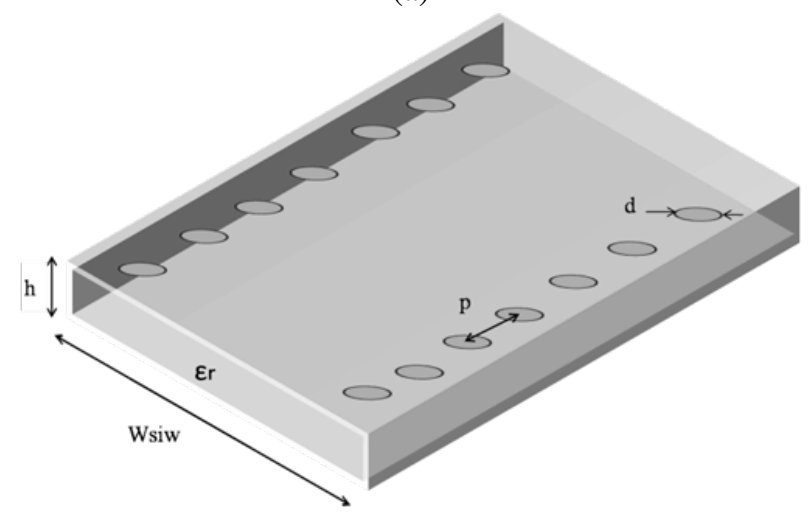

(b)

Figure 1: Structural parameters of: (a) An equivalent rectangular waveguide. (b) A substrate integrated waveguide. 


\section{The equivalent width of the SIW}

A substrate integrated waveguide and an equivalent rectangular waveguide are shown in figure 1. Several formulas linking both widths are presented in the literature. In this section, we will summarize the most popular formulas. The most simple is given by equation 1 . The Boundary IntegralResonant Mode Expansion (BI-RME) method [5, 6], combined with Floquet's theory, is used to find the propagation constant of the structure. The precision of (1) is $5 \%$ according to [1].

$$
W_{S I W}=W_{e q}-\frac{d^{2}}{0.95 p}
$$

Equation 2 gives a recent formula [2], based on the ModeMatching Technique (MMT) [7, 8]. The error of this equation is within $1.2 \%$ of the original data [2].

$$
W_{S I W}=W_{e q}+p\left(0.766 e^{0.4486 d / p}-1.176 e^{-1.241 d / p}\right)
$$

Equivalence on propagation constant and cutoff frequency between a rectangular waveguide and SIW are established using an analytical approach of the Method of Moment (MoM) [9, 10]. An average accuracy of $4 \%$ is assured by the formula given by equation (3) [3].

$$
W_{S I W}=\frac{2 W_{e q}}{\pi} \cot ^{-1}\left(\frac{\pi p}{4 W_{e q}} \log \left(\frac{p}{2 d}\right)\right)
$$

An experimental formula is given for the normalized width of the equivalent waveguide. The relative error of the formula is below $1 \%$ [4].

$$
W_{S I W}=W_{e q} \times\left(x_{1}+\frac{x_{2}}{\frac{p}{d}+\frac{x_{1}+x_{2}-x_{3}}{x_{3}-x_{1}}}\right)
$$

where :

$$
\begin{aligned}
& x_{1}=1.0198+\frac{0.3465}{\frac{W_{e q}}{p}-1.0684} \\
& x_{2}=-0.1183+\frac{1.2729}{\frac{W_{e q}}{p}-1.2010} \\
& x_{3}=1.0082+\frac{0.9163}{\frac{W_{e q}}{p}-2152}
\end{aligned}
$$

\section{Method of analysis}

In this work, we will not dwell on the detailed mathematic formulation of FDFD technique; we will only give some outline of the method. We take advantage of periodicity of SIW structure to restrict the analysis to a single period. A unit cell of SIW with geometrical parameters is shown in figure 2. This structure is analyzed with Finite Difference Frequency Domain (FDFD) method [11, 12], for determining the propagation characteristics. Electric and magnetic fields for the periodic structure can be expressed as given in equation (5), according to the Floquet's theorem [12].

$$
G(x, y, z)=g(x, y, z) e^{-\gamma z}
$$

where $\gamma$ is the propagation constant $(\gamma=\alpha+\mathrm{j} \beta), G(x, y, z)$ is the electric (magnetic) field, and $g(x, y, z)$ represents the periodic function of the electric (magnetic) field. to model this kind of structure, boundary conditions must be applied. We assumed that the upper and bottom planes are perfect electric conductors (PECs) and we used a perfectly matched layer absorbing boundary conditions (PML ABCs) for the lateral walls.

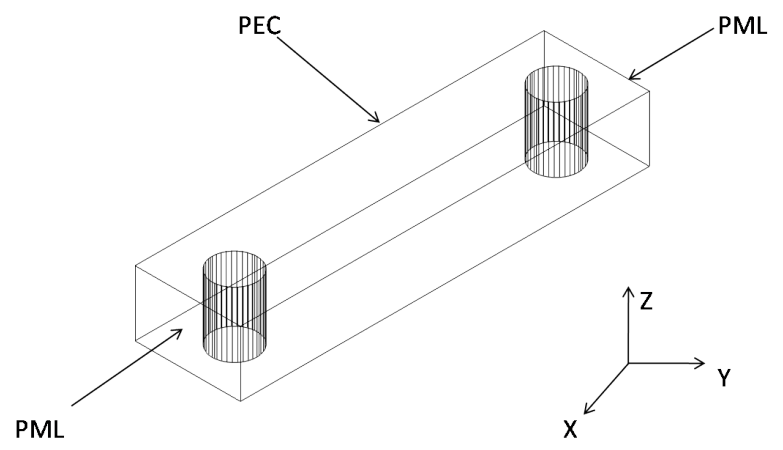

Figure 2: Top view of a unit cell of periodic SIW.

By applying these boundary conditions, Maxwell's curl equations can be expressed in a split form, where each component of the electromagnetic field is split into two parts, so the six field components give twelve sub-components in Cartesian coordinates [13].

$$
\begin{array}{r}
\nabla \times E=-j \omega \mu H \\
\nabla \times H=j \omega \epsilon E+J
\end{array}
$$

The longitudinal components on the Z-axis, $e z$ and $h z$, are eliminated by combining the electromagnetic fields with a split form.

$$
\begin{gathered}
j \omega \mu h_{x y}+\sigma_{y}^{*}(x) h_{x y}=-\frac{\partial\left(e_{z x}+e_{z y}\right)}{\partial y} \\
j \omega \mu h_{x z}=\frac{\partial\left(e_{y z}+e_{y x}\right)}{\partial z}-\gamma\left(e_{y z}+e_{y x}\right) \\
j \omega \mu h_{y z}=-\frac{\partial\left(e_{x y}+e_{x z}\right)}{\partial z}+\gamma\left(e_{x y}+e_{x z}\right) \\
j \omega \mu h_{y x}+\sigma_{x}^{*}(y) h_{y x}=\frac{\partial\left(e_{z x}+e_{z y}\right)}{\partial x} \\
j \omega \mu h_{z x}+\sigma_{x}^{*}(z) h_{z x}=-\frac{\partial\left(e_{y z}+e_{y x}\right)}{\partial x} \\
j \omega \mu h_{z y}+\sigma_{y}^{*}(z) h_{z y}=\frac{\partial\left(e_{x y}+e_{x z}\right)}{\partial y} \\
j \omega \epsilon_{x} e_{x y}+\sigma_{y}(x) e_{x y}=\frac{\partial\left(h_{z x}+h_{z y}\right)}{\partial y} \\
j \omega \epsilon_{y} e_{y x}+\sigma_{x}(y) e_{y x}=-\frac{\partial\left(h_{z x}+h_{z y}\right)}{\partial x} \\
j \omega \epsilon_{x} e_{x z}+\sigma_{z}(x) e_{x z}=\frac{\partial\left(h_{y z}+h_{y x}\right)}{\partial z}+\gamma\left(h_{y z}+h_{y x}\right) \\
+\sigma_{z}(y) e_{y z}=\frac{\partial . \mathrm{b})}{2 . \mathrm{g}}
\end{gathered}
$$




$$
\begin{aligned}
j \omega \epsilon_{z} e_{z x}+\sigma_{x}(z) e_{z x} & =\frac{\partial\left(h_{y z}+h_{y x}\right)}{\partial x} \\
j \omega \epsilon_{z} e_{z y}+\sigma_{y}(z) e_{z y} & =-\frac{\partial\left(h_{x y}+h_{x z}\right)}{\partial y}
\end{aligned}
$$

Where $\sigma$ and $\sigma^{*}$ denote, respectively the electric and magnetic loss. The number of unknown variables is then reduced and the problem can be simplified to a standard matrix eigenvalue problem.

$$
C x=\gamma x
$$

The roots of an eigenvalue equation are the propagation constants for a given frequency. For more details about FDFD algorithm and the problem formulation, the reader will find a full description, including every formula required for Matlab modelling, in reference [13]. The used idea is to vary $W$ siw/Weq ratio according to $d / p$ ratio, where the cutoff frequency and the relative error are calculated for each combination. For reasons of precision, we only choose the combinations associated with a relative error less than $1 \%$. We note that via diameter to via pitch ratio should be between 0.5 and 0.8 to keep the attenuation constant due to radiation loss smaller than $1 \mathrm{~dB} / \mathrm{m}$ [14]. The structure under consideration is operating on $\mathrm{X}$-band with a cutoff frequency $f_{c}=6.653 \mathrm{GHz}$, a relative permittivity $\epsilon_{r}$ of 2.33 and 10 pairs of via holes (about a half wavelength). In figure $3, W$ siw/Weq ratio versus $d / p$ ratio is presented. A least

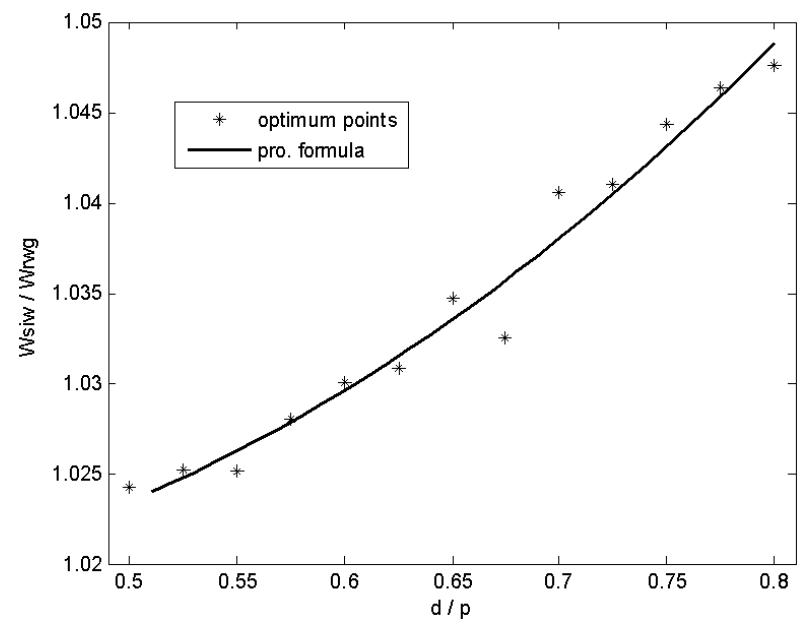

Figure 3: Optimum ratios giving the closest cutoff frequency for $\mathrm{fc}=6.653 \mathrm{GHz}$ and $\epsilon_{r}=2.33$

square approach is used to approximate this curve by the formula (10). Good accuracy of the formula is obtained; the relative error is about $1 \%$.

$$
W_{S I W}=W_{e q} \times\left(0.1172\left(\frac{d}{p}\right)^{2}-0.068\left(\frac{d}{p}\right)+1.0282\right)
$$

\section{Results}

To test the accuracy of this formula, a substrate integrated waveguide containing 20 pairs of via holes is designed and analyzed using the HFSS software. The width of the guide is calculated by the formula 5 and by the formulas reported in [1]- [4]. To get a cutoff frequency closest to the practical cutoff frequency of a rectangular waveguide, an optimum ratio $d / p$ corresponding to the lowest relative error is chosen and in order to cover a large band, this procedure is repeated for many frequencies (from the R-band to the Eband). Figure 4 shows the relative errors obtained. We can observe that the accuracy of formula 5 is comparable with those of the formulas presented in [1]- [4] and our formula presents the weakest relative error. However, formula 3 is less precise than the other formulas. Dispersion characteristics of SIW structure are investigated in this section. For different substrates and different values of frequency band and $d / p$ ratio. The return loss of each guide is obtained with the commercial simulation software HFSS.

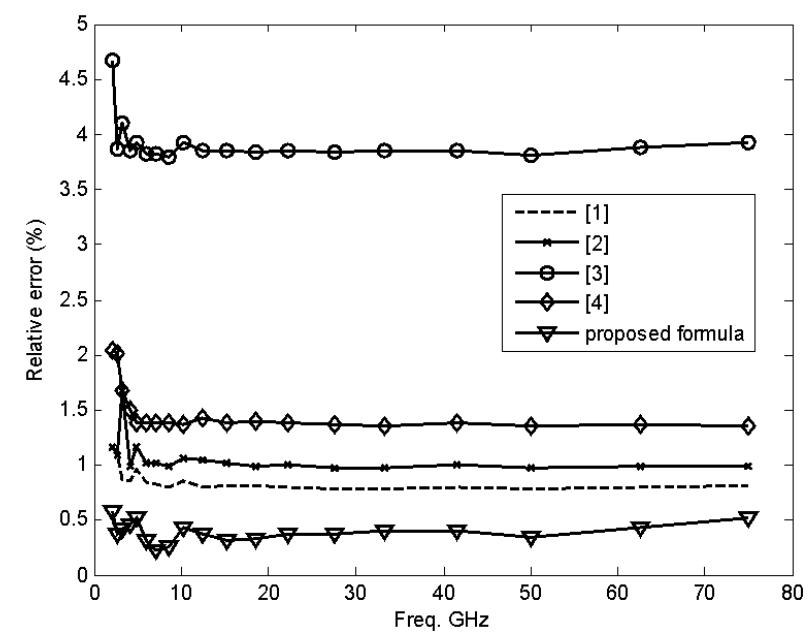

Figure 4: Relative error of different formulas

The first example refers to a SIW operating in X-band at $10 \mathrm{GHz}$. We used an RT/Duroid 5870 dielectric substrate with $\epsilon_{r}=2.33$ and $\operatorname{tg} \delta=0.0012$. The SIW dimensions are: $W e q=14.97 \mathrm{~mm}, p=1 \mathrm{~mm}, d=0.65 \mathrm{~mm}$ and $h=0.508$ $\mathrm{mm}$. Another SIW structure, operating in $\mathrm{K}$ band (15 to $22 \mathrm{GHz}$ ), was also analyzed in the second example using Neltec NH9350 dielectric substrate with $\epsilon_{r}=3.5$ Dimensions of the SIW are: Weq=6.86 $\mathrm{mm}, p=1 \mathrm{~mm}, d=0.8 \mathrm{~mm}$ and $h=0.508 \mathrm{~mm}$. The last test is with a waveguide operating on 22 to $33 \mathrm{GHz}$ band charged with Mica substrate, $\epsilon_{r}=5.7$. Optimum width is $W e q=2.50 \mathrm{~mm}$ for $p=1 \mathrm{~mm}$, $d=0.8 \mathrm{~mm}$ and $h=0.508 \mathrm{~mm}$. Figure 5 shows the return loss of the structure for different widths calculated from different formulas. It can be observed that the return loss is lower than $-20 \mathrm{~dB}$ over the entire frequency band, results are very close. However, the picks of the return loss are phase shifted in frequency from one guide to another due to the difference of the width between the guides. For the figure 5 (a) especially in relatively high frequency, the 


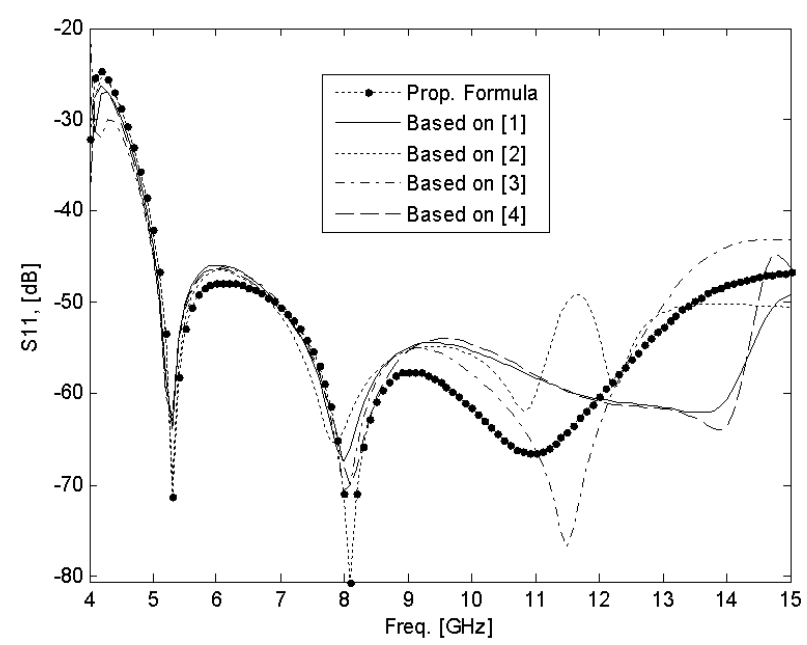

(a)

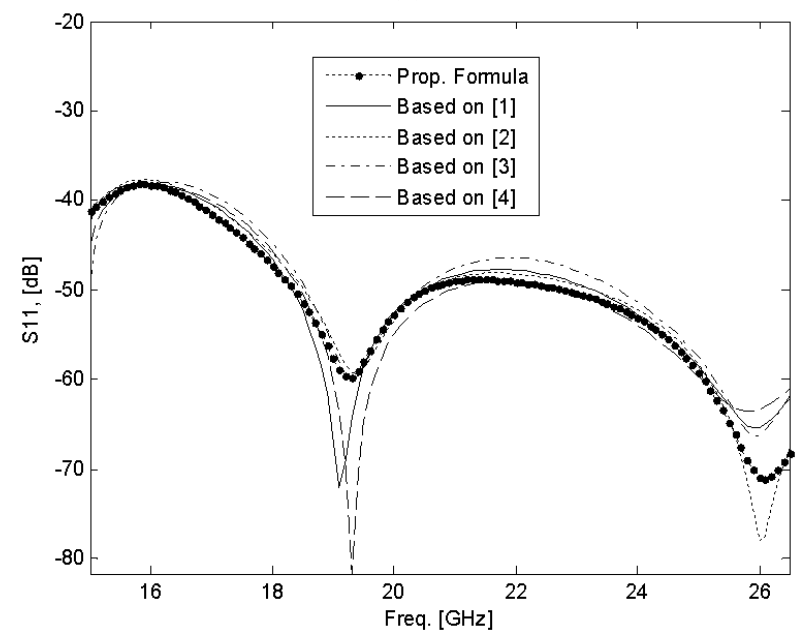

(b)

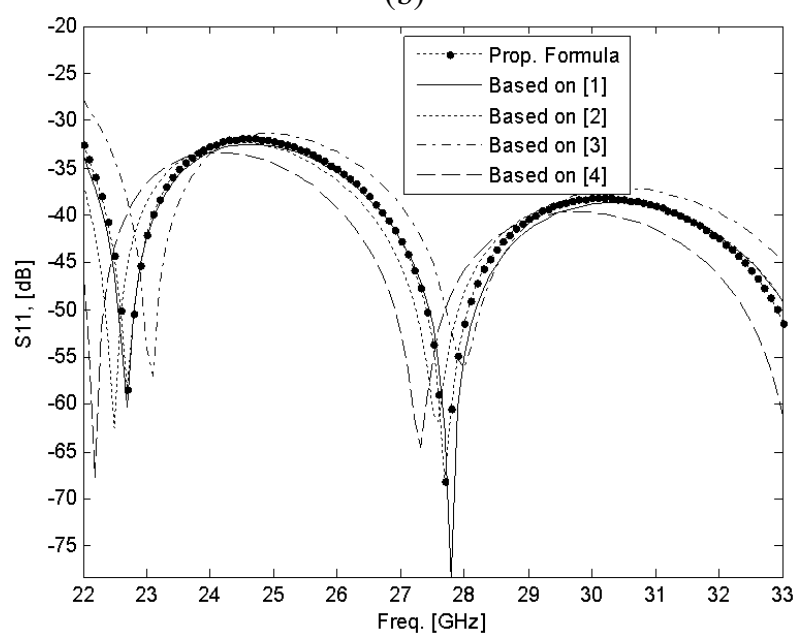

(c)

Figure 5: Reflection coefficient of a SIW obtained by HFSS for: (a) $\epsilon_{r}=2.33$, Weq $=14.97 \mathrm{~mm}, \mathrm{p}=1 \mathrm{~mm}, \mathrm{~d}=0.65 \mathrm{~mm}$, $\mathrm{h}=0.508 \mathrm{~mm}$. (b) $\epsilon_{r}=3.5$, Weq $=6.86 \mathrm{~mm}, \mathrm{p}=1 \mathrm{~mm}, \mathrm{~d}=0.8$ $\mathrm{mm}, \mathrm{h}=0.508 \mathrm{~mm}$. (c) $\epsilon_{r}=5.7$, Weq $=2.50 \mathrm{~mm}, \mathrm{p}=1 \mathrm{~mm}$, $\mathrm{d}=0.8 \mathrm{~mm}, \mathrm{~h}=0.508 \mathrm{~mm}$. impact of the difference between the widths is important whose slight error considerably affects the return loss. In term of return loss, the proposed formula has a similar accuracy compared to other formulas.

Figure 6 shows the simulated phase constant for the first mode TE10 compared to the measured phase constant reported in [1] and [15]. From these figures, we see that the SIW structure designed by using the proposed formula (5) has the same propagation characteristics of a practical waveguide. Proposed formula presents a good precision.

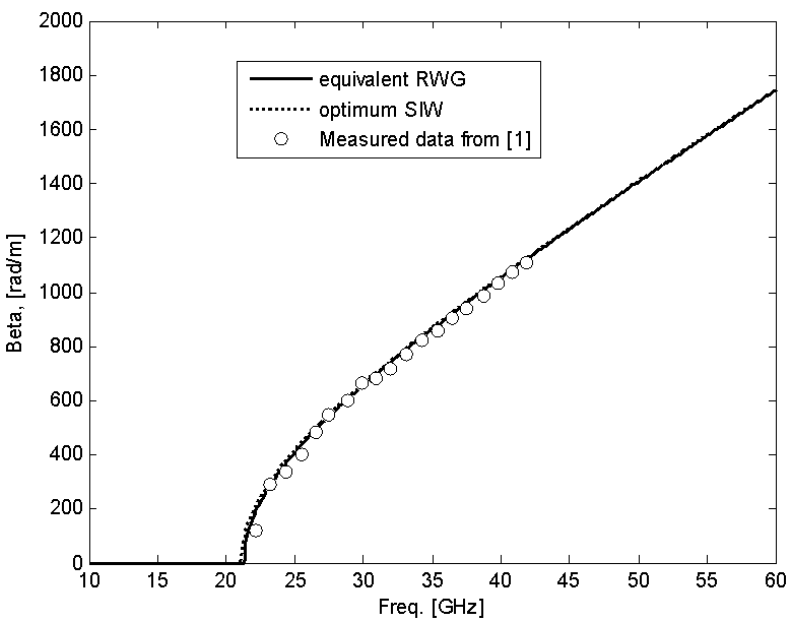

(a)

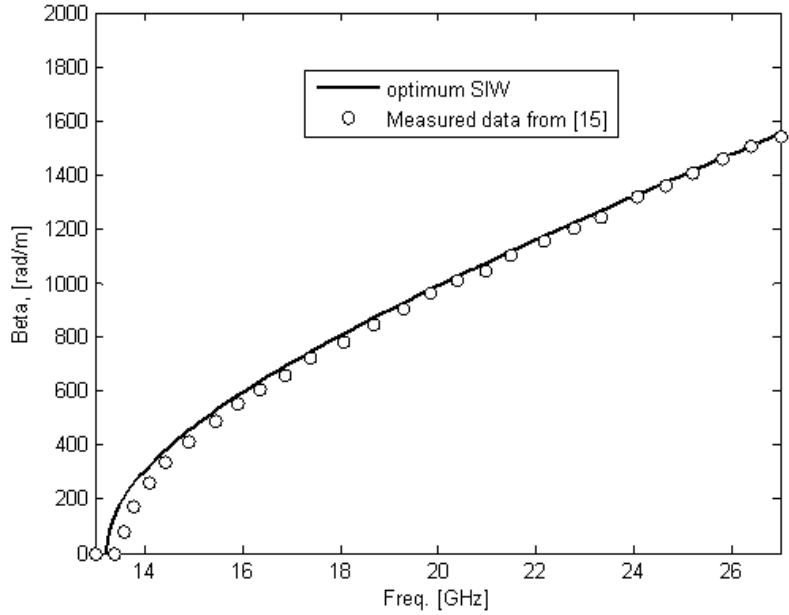

(b)

Figure 6: Propagation constant of fundamental mode TE10 versus frequency for: (a) $\epsilon_{r}=2.2, \mathrm{~d}=0.8 \mathrm{~mm}, \mathrm{p}=1.5 \mathrm{~mm}$, Weq $=4.8 \mathrm{~mm}$. (b) $\epsilon_{r}=9.9, \mathrm{~d}=0.635 \mathrm{~mm}, \mathrm{p}=1.016 \mathrm{~mm}$, Weq $=3.97 \mathrm{~mm}$.

\section{Conclusions}

The FDFD method is used to obtain the propagation characteristics of a substrate integrated waveguide. Based on the FDFD technique, an approach was used to find a formula for calculating the optimal width of the guide. The new formula permits a direct calculation of optimum width of SIW, without resorting to an iterative method or solving 
a high-order polynomial. The accuracy of the new formula is investigated by the design of two guides whose propagation constants are compared with measurement results. Obtained results proved that the optimum width calculated by the new formula gives similar propagation characteristics as the practical waveguide.

\section{References}

[1] Y. Cassivi, L. Perregrini, P. Arcioni, M. Bressan, K. Wu, G. Conciauro, Dispersion characteristics of substrate integrated rectangular waveguide, IEEE Microw. Wireless Compon. Lett. 12: 333-335, 2002.

[2] Z. Kordiboroujeni, J. Bornemann, Designing the Width of Substrate Integrated Waveguide Structures, IEEE Microwave And Wireless Compon. Lett. 23: 518-520, 2013.

[3] W. Che, K. Deng, D. Wang, Y.L. Chow, Analytical equivalence between substrate-integrated waveguide and rectangular waveguide, IET Microw. Antennas Propag. 2: 35-41, 2008.

[4] L. Yan, W. Hong, G. Hua, J. Chen, K. Wu, Simulation and experiment on SIW slot array antennas, IEEE Microw. Wireless Compon. Lett. 14: 446-448, 2004.

[5] G. Conciauro, M. Guglielmi, R. Sorrentino, Advance Modal Analysis, CAD Techniques for Waveguide Components and Filters, Wiley IEEE Press, New York, pp.161-175, 1999.

[6] M. Bozzi, L. Perregrini, K. Wu, Modeling of Conductor, Dielectric and Radiation Losses in Substrate Integrated Waveguide by the Boundary Integral-Resonant Mode Expansion Method, IEEE Transactions on Microwave Theory and Techniques. 56:3153-3161, 2008.

[7] J. Bornemann, F. Taringou, Z. Kordiboroujeni, A mode-matching approach for the analysis and design of substrate-integrated waveguide components, Freq.J. RF/Microw. Engr. Photon. 65: 287-292, 2011.

[8] M. Casaletti, G. Valerio, R. Sauleau, M. Albani, Mode-Matching Analysis of Lossy SIW Devices, IEEE Trans. Microwave Theory Tech. 64: 4126-4137, 2016.

[9] R. F. Harrington, Field computation by moment methods, Wiley IEEE Press, New York, 1993.

[10] E. Arnieri, G. Amendola, Method of Moments Analysis of Slotted Substrate Integrated Waveguide Arrays, IEEE Transactions on Antennas and Propagation. 59: 1148-1154, 2011.

[11] C. L. Da, S. S. Sobrinho, P. P. Silvester, Analysis of a infinite array of rectangular anistropic dielectric waveguide using finit-diffrence method, IEEE Trans. Microwave Theory. 40: 1021-1025, 1992.
[12] A. C. Cangellaris, M. Gribbons, G. Sohos, A hybrid spectral /FDTD method for the electromagnetic analysis of guided waves in periodic structures, IEEE Microwave Theory and Techniques Society. 3: 375-377, 1993.

[13] F. Xu,Y. Zhang, W. Hong, K. Wu, T. J. Cui, Finitedifference frequency-domain algorithm for modeling guided-wave properties of substrate integrated waveguide, IEEE Transactions on Microwave Theory and Techniques. 51: 2221-2227, 2003.

[14] S. S. Karimabadi, A. R. Attari, Circuit analysis and optimisation of SIW branch line coupler with improved modelling of small metallic post in FDTD, IET Microwaves, Antennas Propagation. 11: 617624, 2017.

[15] D. Deslandes, K. Wu, Accurate modeling, wave mechanisms, and design considerations of a substrate integrated waveguide, IEEE Trans. Microw. Theory Tech. MTT-54: 2516-2526, 2006. 